\title{
Naringin inhibits vascular endothelial cell apoptosis via endoplasmic reticulum stress- and mitochondrial-mediated pathways and promotes intraosseous angiogenesis in ovariectomized rats
}

\author{
WEN-JI SHANGGUAN ${ }^{1 *}$, YUE-HUI ZHANG ${ }^{2 *}$, ZHAN-CHUN LI ${ }^{3}$, LU-MIN TANG $^{1}$, JIANG SHAO ${ }^{2}$ and HE LI ${ }^{1}$ \\ ${ }^{1}$ Department of Traditional Chinese Medicine, Renji Hospital, School of Medicine, \\ Shanghai Jiao Tong University, Shanghai 200127; ${ }^{2}$ Department of Orthopedic Surgery, Xin Hua Hospital \\ Affiliated to Shanghai Jiao Tong University School of Medicine, Shanghai 200092; ${ }^{3}$ Department of Orthopedics, \\ Renji Hospital, School of Medicine, Shanghai Jiao Tong University, Shanghai 200127, P.R. China
}

Received July 27, 2016; Accepted September 6, 2017

DOI: $10.3892 /$ ijmm.2017.3160

\begin{abstract}
In this study, to investigate the effects of naringin on vascular endothelial cell (VEC) function, proliferation, apoptosis, and angiogenesis, rat VECs were cultured in vitro and randomly divided into four groups: control, serum-starved, low-concentration naringin treatment, and high-concentration naringin treatment. MTT assay was used to detect cell proliferation while Hoechst 33258 staining and flow cytometry were used to detect apoptosis. Changes in the expression of apoptosis-associated proteins [GRP78, CHOP, caspase-12, and cytochrome $c$ (Cyt.c)] were detected using western blotting. JC-1 staining was employed to detect changes in mitochondrial membrane potential. Intracellular caspase-3, -8 , and -9 activity was determined by spectrophotometry. ELISA was used to detect endothelin (ET), and a Griess assay was used to detect changes in the expression of nitric oxide (NO) in culture medium. The study further divided an ovariectomized (OVX) rat model of osteoporosis randomly into four groups: OVX, sham-operated, low-concentration naringin treatment $(100 \mathrm{mg} / \mathrm{kg})$, and high-concentration
\end{abstract}

Correspondence to: Dr Jiang Shao, Department of Orthopedic Surgery, Xin Hua Hospital Affiliated to Shanghai Jiao Tong University School of Medicine, 1665 Kongjiang Road, Shanghai 200092, P.R. China

E-mail: shaojxh@126.com

Dr He Li, Department of Traditional Chinese Medicine, Renji Hospital, School of Medicine, Shanghai Jiao Tong University, 160 Pujian Road, Shanghai 200127, P.R. China

E-mail: lihe1972@hotmail.com

\section{${ }^{*}$ Contributed equally}

Key words: naringin, vascular endothelial cells, apoptosis, angiogenesis, osteoporosis naringin treatment $(200 \mathrm{mg} / \mathrm{kg})$. After 3 months of treatment, changes in serum ET and NO expression, bone mineral density (BMD), and microvessel density of the distal femur (using CD34 labeling of VECs) were determined. At each concentration, naringin promoted VEC proliferation in a time- and dose-dependent manner. Naringin also significantly reduced serum starvation-induced apoptosis in endothelial cells, inhibited the expression of GRP78, CHOP, caspase-12, and Cyt.c proteins, and reduced mitochondrial membrane potential as well as reduced the activities of caspase-3 and -9 . Furthermore, naringin suppressed ET in vitro and in vivo while enhancing NO synthesis. Distal femoral microvascular density assessment showed that the naringin treatment groups had a significantly higher number of microvessels than the OVX group, and that microvascular density was positively correlated with BMD. In summary, naringin inhibits apoptosis in VECs by blocking the endoplasmic reticulum (ER) stress- and mitochondrial-mediated pathways. Naringin also regulates endothelial cell function and promotes angiogenesis to exert its anti-osteoporotic effect.

\section{Introduction}

Osteoporosis (OP) is a systemic metabolic bone disease characterized by an increase in bone fragility and susceptibility to bone fracture. It not only reduces the quality of life of elderly individuals, but also causes significant financial burden to patients' families and society. Despite the fact that OP has developed into a serious social issue that has gained extensive attention worldwide (1), the pathogenesis of OP remains unclear.

Blood vessels are an important factor in the process of bone formation, and angiogenesis plays an important role in maintaining the dynamic balance between bone regeneration and resorption (2). Exogenous vascular endothelial growth factor (VEGF) has been shown to enhance fracture repair in a mouse model of femoral fracture by promoting angiogenesis, ossification, and bone turnover (3). Similarly, angiogenesis is 
an important feature of fracture repair, where an adequate blood supply is the basis of successful bone regeneration (4). Furthermore, inhibition of angiogenesis has been shown to inhibit fracture repair (5). The incidence of postmenopausal $\mathrm{OP}$ is associated with a reduction in bone marrow microvessels, and the promotion of angiogenesis exhibits anti-osteoporotic effects (6). Therefore, in view of the importance of angiogenesis in the growth and development of bone, it may be closely associated with OP (7-10).

Vascular endothelial cells (VECs) are simple squamous cells that exhibit selective permeability, regulate vascular tone, promote blood capillary formation, and exhibit other important physiological functions. Several studies have shown that apoptosis in VECs is closely associated with endoplasmic reticulum (ER) stress and mitochondrial depolarization, and that inhibition of apoptosis can protect the vascular endothelium $(11,12)$. In addition, VECs secrete various vasoactive substances that regulate vascular function such as endothelin (ET) and nitric oxide (NO), which can further affect bone metabolism. Prispy et al (13) found that reduction in metaphyseal bone mass is associated with decreased bone marrow blood flow in elderly patients, and is induced by inhibition of endothelium-dependent vasodilation. Furthermore, physical exercise-induced increase in bone mass was shown to be associated with enhanced endothelium-dependent vasodilation via the NO synthase signaling pathway (14).

Naringin, a flavonoid compound, is the main active ingredient in the traditional Chinese medicinal plant Drynaria, and exerts various biological and pharmacological effects (15). Drynaria is widely used in the treatment of OP, although its mechanism remains unclear (9). Studies have shown that naringin promotes the differentiation and proliferation of various types of cells (16-18), and was found to improve bone mass in an osteoporotic rat model (19). Naringin also promoted osteoclast apoptosis through the mitochondrial-mediated apoptotic pathway, thereby inhibiting bone loss in an ovariectomized (OVX) rat model of OP and exhibiting anti-osteoporotic pharmacological activity (20). However, its effects on VECs and angiogenesis remain unclear. The present study explored the effects of naringin on the function, proliferation, and apoptosis of VECs, as well as on angiogenesis and its mechanisms, to provide a theoretical basis for the clinical application of naringin in the treatment of OP.

\section{Materials and methods}

\section{In vitro experiments}

Culture of rat VECs. All protocols were approved by the Renji Hospital Animal Care and Ethics Committee. Lung tissue was aseptically excised from 1-day-old newborn Sprague-Dawley (SD) rats and rinsed with phosphate-buffered saline (PBS) to remove the pleura. Tissue was trimmed into $1-\mathrm{mm}^{3}$ tissue blocks, cultured in Dulbecco's modified Eagle's medium (DMEM) low-glucose medium supplemented with $10 \%$ serum in $6-\mathrm{cm}$ culture dishes, and incubated at $37^{\circ} \mathrm{C}$ and $5 \% \mathrm{CO}_{2}$ for about 3-5 days until cells emerged from the tissue edges. Cells were digested with $0.25 \%$ trypsin, filtered to remove tissue, and further cultured for 1 week. After that, cells were digested and sub-cultured until the fifth passage before use.
MTT cell proliferation assay. MTT assay was performed as described previously (18). Briefly, VECs were seeded at a density of $1 \times 10^{4}$ cells/well into a 96-well plate. After $12 \mathrm{~h}$ of culture, cells were treated with $10 \%$ fetal bovine serum (FBS) containing 0, 1, 10, 50, 100, or $200 \mu \mathrm{g} / \mathrm{ml}$ naringin (Sigma-Aldrich, St. Louis, MO, USA) for 12, 24, 36, 48, 60, 72, or 96 h. Next, cells were collected and an MTT assay was performed according to the manufacturer's instructions (Beyotime Institute of Biotechnology, Shanghai, China).

Hoechst 33258 staining. VECs were randomly divided into four groups: control (10\% FBS), serum starvation (0\% FBS), low-concentration treatment $(0 \% \mathrm{FBS}+10 \mu \mathrm{g} / \mathrm{ml}$ naringin), and high-concentration treatment ( $0 \% \mathrm{FBS}+100 \mu \mathrm{g} / \mathrm{ml}$ naringin). Serum-free starvation culture conditions were established by growing cells to $80-90 \%$ confluence before discarding the medium, washing cells twice with PBS, and culturing in FBS-free DMEM. This cell preparation procedure was repeated for subsequent cytological assays.

For Hoechst 33258 staining, cells were seeded at $1 \times 10^{5}$ cells/well into a 6 -well plate. Cells were treated for $24 \mathrm{~h}$, and cellular morphological changes were observed by inverted phase contrast microscopy. Culture medium was removed and Hoechst 33258 staining solution (Beyotime Institute of Biotechnology) was added to the cells. Morphological nuclear changes were observed under fluorescence microscopy.

Detection of apoptosis by flow cytometry. Cells were seeded at $2 \times 10^{5}$ cells/well into $6-\mathrm{cm}$ culture dishes and treated as described above for 24 or $48 \mathrm{~h}$ prior to digestion with $0.05 \%$ trypsin-EDTA to harvest the cells. Cells were then subjected to Annexin V/propidium iodide (PI) staining and flow cytometry was performed according to the manufacturer's instructions (BD Pharmingen, San Diego, CA, USA). Apoptotic cells were counted and represented as a percentage of the total cell count.

Detection of proteins associated with apoptosis by western blotting. Cells were seeded at $5 \times 10^{5}$ cells/well into $6-\mathrm{cm}$ culture dishes and treated for $24 \mathrm{~h}$ as described above, after which $100 \mu 12 \mathrm{X}$ SDS cell lysis solution was added per dish. Cellular proteins were then harvested and stored at $-70^{\circ} \mathrm{C}$. A mitochondrial and cytosolic protein preparation kit (Applygen Technologies, Inc., Beijing, China) was used to extract cytoplasmic proteins for the detection of cytochrome $c$ (Cyt.c). After measurement of protein concentrations, $40 \mu \mathrm{g}$ of protein per sample was subjected to electrophoresis and subsequently transferred to a nitrocellulose membrane (Bio-Rad, Berkeley, CA, USA). The membrane was blocked using skimmed milk and incubated overnight at $4{ }^{\circ} \mathrm{C}$ with primary antibodies for GRP78 (sc-13968), CHOP (sc-7351), caspase-12 (sc-5627), or Cyt.c (sc-13561) (Santa Cruz Biotechnology, Inc., Santa Cruz, CA, USA) at 1:100 dilution, or with antibody for GAPDH (sc-47724; Santa Cruz Biotechnology, Inc.) at a 1:750 dilution. Next, the membrane was washed and incubated at room temperature for $2 \mathrm{~h}$ with secondary antibodies (cat. no. A0192; Beyotime Institute of Biotechnology), followed by enhanced chemiluminescence (ECL) autoradiography. Band intensity was measured using an ImageMaster ${ }^{\mathrm{TM}}$ VDS video documentation system (Amersham Biosciences Inc., Piscataway, NJ) and represented as fold-change relative to the GAPDH control.

Detection of changes in mitochondrial membrane potential by JC-1 staining. Cells were seeded at $1 \times 10^{5}$ cells/well into 6 -well plates and treated for $24 \mathrm{~h}$. JC-1 probe (Beyotime 
Institute of Biotechnology) was added to detect changes in mitochondrial membrane potential as described in our previous study (21).

Detection of caspase-3, -8 , and -9 activities. Cells were seeded at $5 \times 10^{5}$ cells/well into $6-\mathrm{cm}$ culture dishes, and harvested after $24 \mathrm{~h}$ of treatment. Caspase- $3,-8$, and -9 activities were detected using a caspase- $3,-8$, and -9 activity assay kit (Beyotime Institute of Biotechnology), and OD at $405 \mathrm{~nm}$ was measured using a microplate spectrophotometer (BioTek, Winooski, VT, USA). The caspase activity of cells in each treatment group was represented as fold-change in absorbance relative to control.

Detection of ET and NO in culture medium. Cells were seeded at $5 \times 10^{5}$ cells/well into 6 -cm culture dishes and treated for 24 or $48 \mathrm{~h}$ before $100 \mu \mathrm{l}$ of the supernatant was collected from each well and centrifuged to remove impurities and cell debris. ET was detected by ELISA. A standard curve was obtained via serial dilution of standards included in the assay kit (Beyotime Institute of Biotechnology). Each sample was aliquoted into a microtiter plate and OD at $450 \mathrm{~nm}$ was measured. NO was detected using a Griess assay (Beyotime Institute of Biotechnology), carried out in accordance with the manufacturer's instructions and with OD measured at $540 \mathrm{~nm}$ using a microplate spectrophotometer.

\section{In vivo experiments}

Postmenopausal osteoporotic rat model grouping and drug treatment. Six-month-old female SD rats (body weight, $220 \pm 8 \mathrm{~g}$ ) were provided by the Experimental Animal Center of Renji Hospital. The experimental animals received humane care, and the study protocol conformed to the guidelines of the Renji Hospital Ethics Committee. Forty-eight rats were randomly divided into four groups ( $\mathrm{n}=12$ per group): OVX, sham, high-concentration naringin treatment $(200 \mathrm{mg} / \mathrm{kg})$, and low-concentration naringin treatment $(100 \mathrm{mg} / \mathrm{kg})$. After rats were anesthetized via an intraperitoneal injection of ketamine $(75 \mathrm{mg} / \mathrm{kg})$, bilateral incisions were made at the lower back into the abdominal cavity. The incision wounds were washed and sutured after removing both ovaries. For the sham group, fat of equivalent weight to an ovary was excised from the vicinity of each ovary. On the third day after ovariectomy, rats in the high- and low-concentration treatment groups were administered naringin solution by daily oral gavage. Rats in the OVX and sham groups were administered physiological saline solution ( $6 \mathrm{ml} / \mathrm{kg}$ daily) by oral gavage. After 3 months of treatment, rats were sacrificed by anesthesia with an intraperitoneal injection of sodium pentobarbital $(40 \mathrm{mg} / \mathrm{kg})$.

Bone densitometry. Dual-energy X-ray absorptiometry (DXA; GE Healthcare, Piscataway, NJ, USA) was used to measure bone mineral density (BMD) of the fourth lumbar vertebra (L4), and data analyses were carried out using pre-installed software.

Detection of ET and NO in serum. Blood was collected from the abdominal aorta of rats prior to sacrifice and centrifuged. Changes in ET and NO expression were detected in $100 \mu \mathrm{l}$ aliquots of the resulting serum using the method described above.

Bone marrow angiogenesis assay. Distal femur specimens from rats were routinely decalcified and embedded in paraffin. Immunohistochemical staining of VECs for CD34 was

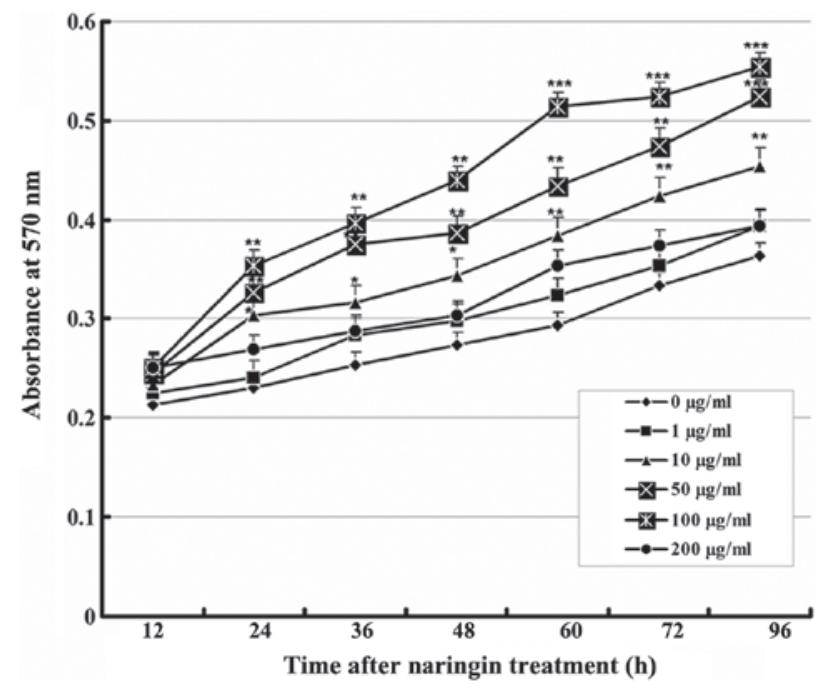

Figure 1. Effect of naringin on the proliferation of VECs. Cells were cultured with vehicle or various concentrations of naringin. At 12, 24, 36, 48, 60,72 , and $96 \mathrm{~h}$ after treatment, absorbance at $570 \mathrm{~nm}$ was measured $(\mathrm{n}=9)$ Comparison with the control group: ${ }^{*} \mathrm{P}<0.05,{ }^{* *} \mathrm{P}<0.01,{ }^{* * *} \mathrm{P}<0.001$. VECs, vascular endothelial cells.

carried out according to the manufacturer's instructions (R\&D Systems, Inc., Minneapolis, MN, USA). Specimens were observed under a microscope at x100 magnification and microvessels in three randomly selected fields of view were counted to obtain an average value (22).

Statistical analysis. The results of each measurement are represented as mean \pm SD and data processing was performed using SPSS v18.0 software (SPSS Inc., Chicago, IL, USA). One-way analysis of variance (ANOVA) was used for comparisons between groups, and $\mathrm{P}<0.05$ was considered statistically significant.

\section{Results}

Effect of naringin on VEC proliferation. The MTT assay results showed that each group treated with a naringin dose of $1-100 \mu \mathrm{g} / \mathrm{ml}$ had a significantly higher cell proliferation rate than the control group, and that the proliferation-promoting effect increased significantly in a concentration-dependent manner up to $100 \mu \mathrm{g} / \mathrm{ml}$. However, the proliferation-promoting effect ceased at $200 \mu \mathrm{g} / \mathrm{ml}$, an effect that may be related to drug toxicity. These results revealed that naringin promoted the proliferation of VECs in a specific time- and dose-dependent manner (Fig. 1).

Effects of naringin on serum starvation-induced apoptosis in VECs. After $24 \mathrm{~h}$, cells in the serum starvation group showed apoptotic morphology including cell rounding and shrinkage, vacuolization, and detachment from the plate (Fig. 2A); Hoechst 33258 staining showed increased chromatin condensation and nuclear fragmentation. However, treatment with a high concentration of naringin effectively suppressed these phenomena (Fig. 2B). After cells were starved for 24 and 48 h, flow cytometry showed that the average apoptosis ratio in the control VECs was only $2.3 \pm 0.8 \%$, but was significantly increased up to $31.1 \pm 1.6$ and $39.6 \pm 1.7 \%$, respectively. The 

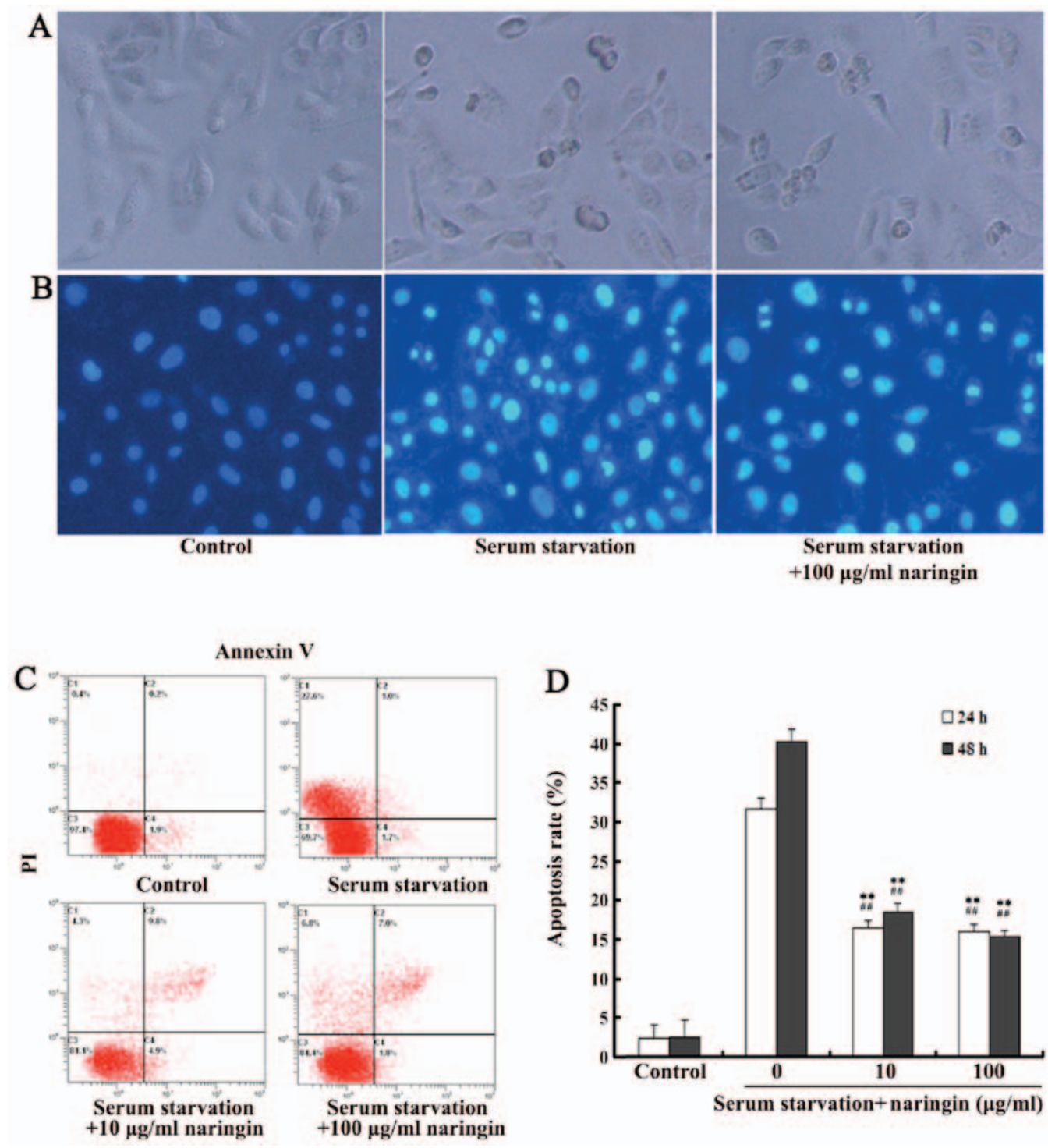

Figure 2. Effects of naringin on starvation-induced apoptosis in VECs. (A and B) Effects of naringin on morphological changes [(A) x200 magnification] and morphological changes in nuclei by Hoechst 33258 staining [(B) x320 magnification] of VEC apoptosis induced by starvation for $24 \mathrm{~h}$. (C) Density plots generated by flow cytometry after Annexin V-FITC and PI double-staining. (D) Effects of naringin on the apoptosis ratio in starvation-induced VECs. Comparison with the serum starvation group: ${ }^{* *} \mathrm{P}<0.01$; comparison with the control group: ${ }^{\# \#} \mathrm{P}<0.01$. VECs, vascular endothelial cells.

apoptosis ratio in both the high- and low-concentration naringin treatment groups was significantly decreased compared to that in the serum starvation group $(\mathrm{P}<0.01$; Fig. $2 \mathrm{C}$ and $\mathrm{D})$.

Effects of naringin on the expression of proteins associated with ER stress-induced apoptosis. After $24 \mathrm{~h}$ of serum starvation, protein expression of GRP78, CHOP, and caspase-12 was increased significantly compared with that noted in the control group. The expression of GRP78 and CHOP in both the high- and low-concentration naringin treatment groups was significantly decreased compared to that in the serum starvation group. Furthermore, caspase-12 expression in the high-concentration treatment group was significantly decreased compared to that in the serum starvation group. Although the expression of caspase-12 was also decreased in the low-concentration treatment group, the difference was statistically insignificant compared with the serum starvation group (Fig. 3A and B). According to our results, the inhibition of these three proteins by naringin did not reach control levels, suggesting that naringin partially inhibits the expression of proteins associated with starvation-induced ER stress.

Effects of naringin on the mitochondrial-mediated apoptotic pathway. After $24 \mathrm{~h}$ of serum starvation, the mitochondrial membrane potential of the serum starvation group was significantly decreased compared with that of the control group (average value of red/green fluorescence intensity, $0.6 \pm 0.2$ vs. $3.6 \pm 0.3, \mathrm{P}<0.01)$. The mitochondrial membrane potential in both the high- and low-concentration naringin treatment groups $(1.5 \pm 0.3$ and $1.8 \pm 0.4$, respectively) was significantly increased compared to that in the serum starvation group (3.6 \pm 0.3$)$ but did not reach control levels, suggesting that naringin partially inhibits the mitochondrial membrane depolarization induced by starvation (Fig. 4A and B). After $24 \mathrm{~h}$ of starvation, Cyt.c protein expression in the cytoplasm was significantly increased, and both the low and high concen- 
A
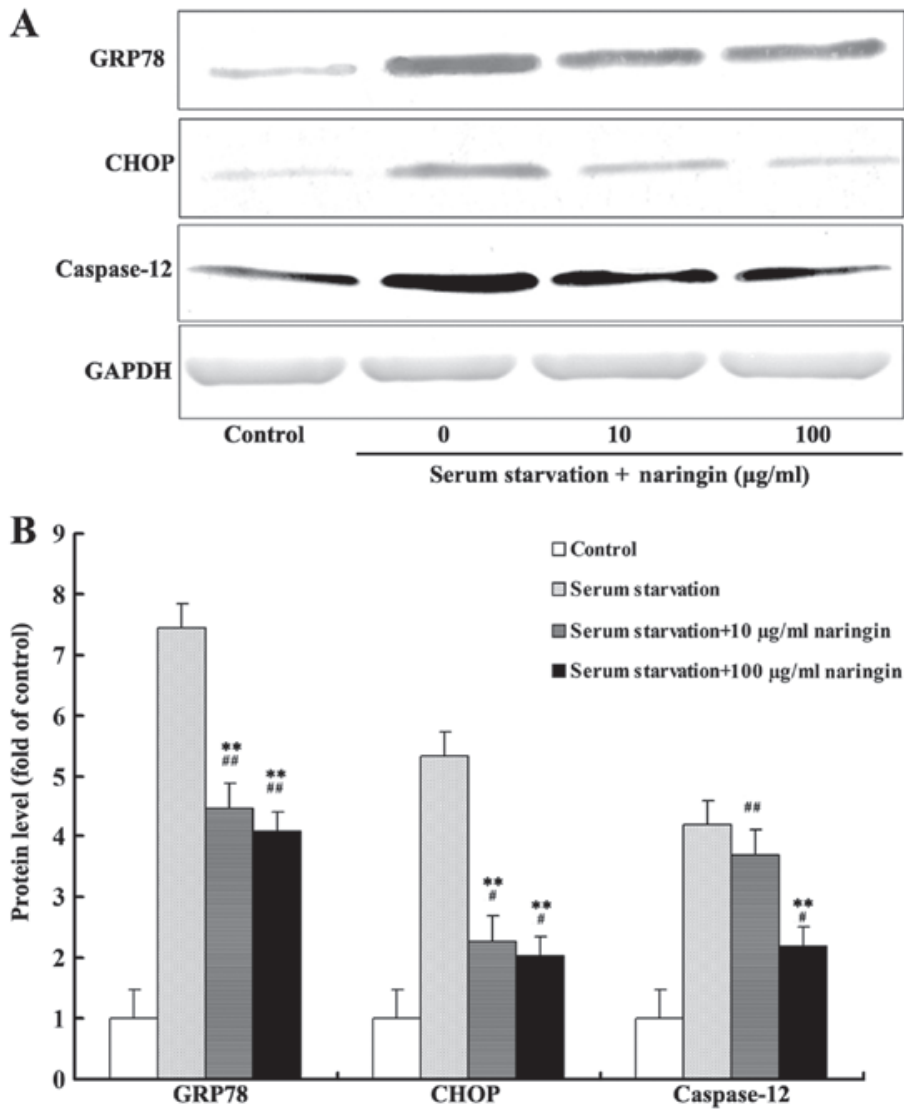

Figure 3. Detection of ER stress marker protein expression (CHOP, GRP78, and caspase-12) by western blotting. (A) Western blotting images showing changes in protein expression after $24 \mathrm{~h}$ of treatment. (B) Semi-quantitative analysis of protein expression levels. Comparison with the serum starvation group: ${ }^{* *} \mathrm{P}<0.01$; comparison with the control group: ${ }^{\#} \mathrm{P}<0.01,{ }^{\# \#} \mathrm{P}<0.01$. ER, endoplasmic reticulum.

trations of naringin significantly inhibited the release of Cyt.c protein into the cytoplasm $(\mathrm{P}<0.01)(\mathrm{Fig} .4 \mathrm{C}$ and $\mathrm{D})$.

Effects of naringin on caspase-3, -8, and -9 activities. Caspase- 3 is an important protein for apoptosis execution, while the activation of caspase-9 plays a key role in the mitochondrial-mediated apoptotic pathway. Our results showed that caspase-3 and -9 activities were significantly increased after $24 \mathrm{~h}$ of starvation, and that both high and low concentrations of naringin significantly inhibited caspase- 3 and -9 activities $(\mathrm{P}<0.05)$. Caspase-8 activity in each treatment group did not change (Fig. 5A).

Effects of naringin on ET and NO expression. After serum starvation, the expression of ET was significantly increased while the expression of NO was decreased in the serum starvation group. Both high and low concentrations of naringin significantly reduced the ratio of ET to NO by inhibiting the expression of ET while enhancing the expression of NO. Differences in ET and NO levels between 24 and $48 \mathrm{~h}$ of starvation and between high and low concentrations of naringin were not statistically significant $(\mathrm{P}>0.05)$. These results indicated that naringin achieved drug efficacy at a low concentration at $24 \mathrm{~h}$, and that a further increase in drug concentration and exposure time did not enhance the effectiveness of the drug (Fig. 5B-D). After OVX rats were administered high and low concentrations of naringin for 3 months, the expression of ET in serum was significantly decreased $(\mathrm{P}<0.05)$ while the expression of $\mathrm{NO}$ was significantly increased compared with the OVX group $(\mathrm{P}<0.05)$. However, the expression levels of both ET and NO did not decrease to the levels observed in the sham group $(\mathrm{P}<0.05)$ (Fig. 6A-C).

Effects of naringin on BMD. BMD of the OVX group was significantly decreased compared to that of the sham group, indicating that the model design was successful. The BMD of the fourth lumbar vertebra (L4) in both the high- and low-concentration naringin treatment groups was significantly increased compared to that of the OVX group after surgery $(\mathrm{P}<0.05)$, but did not increase to the BMD levels of the sham group (Fig. 6D).

Effects of naringin on microvascular angiogenesis. The results of microvascular density analysis (number of microvessels/x100 magnification) showed that microvascular density in both the high- and low-concentration naringin groups was increased significantly compared to that in the OVX group $(\mathrm{P}<0.05)$, although it was lower than the sham group microvascular density (Fig. 6E-H).

\section{Discussion}

VECs and new blood vessels are important factors in the process of bone formation (2). Evidence has shown that postmenopausal OP may be associated with the apoptosis and dysfunction of VECs and a decrease in bone marrow microvessels, and 


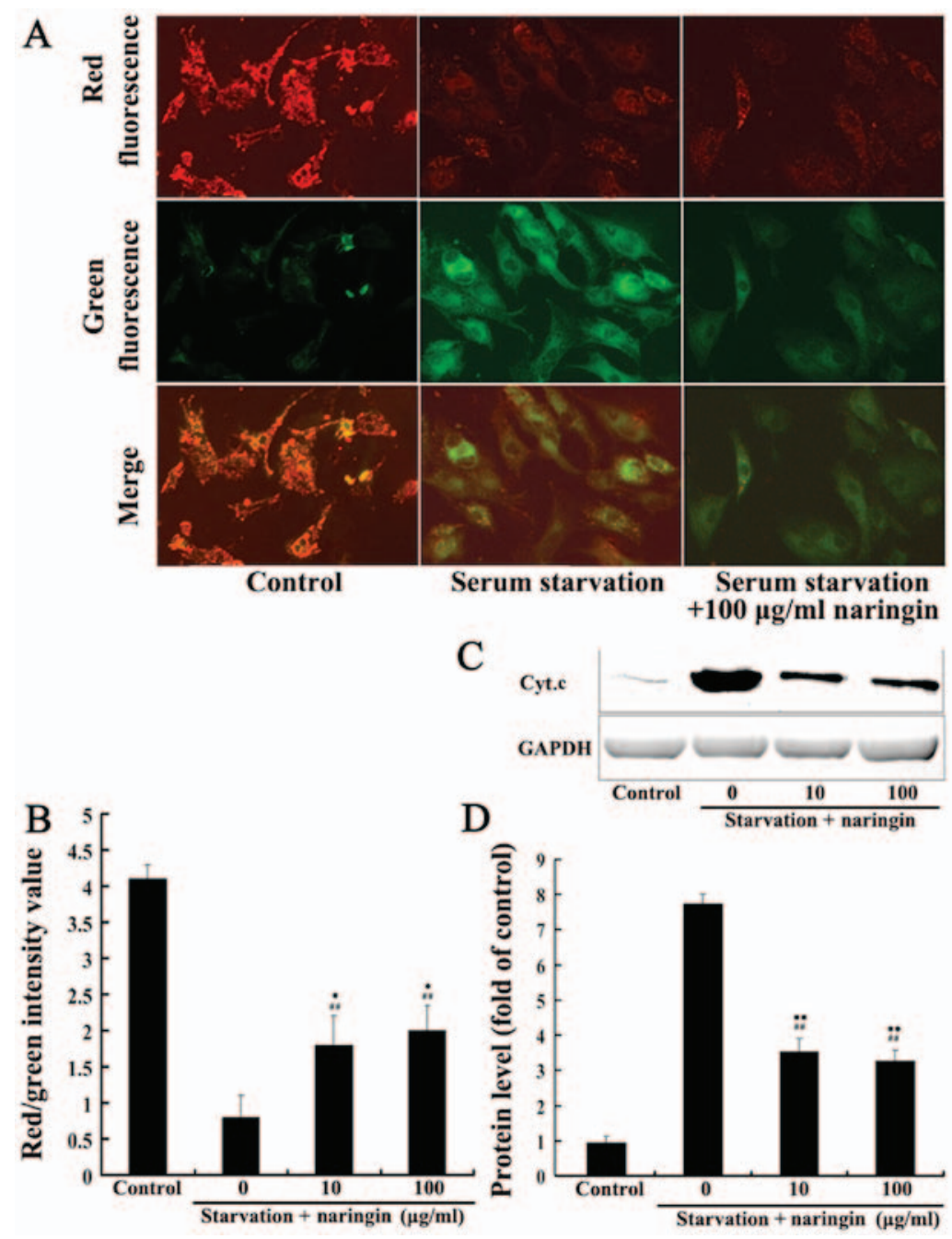

Figure 4. Effects of naringin on the mitochondrial-mediated apoptotic pathway after $24 \mathrm{~h}$ of serum starvation. (A) Images showing JC-1-stained cells. Original magnification, x200. (B) Semi-quantitative analysis of red/green fluorescence intensity value. (C) Images showing the detection of cytoplasmic Cyt.c level by western blotting. (D) Semi-quantitative analysis of cytoplasmic Cyt.c levels. Comparison with the serum starvation group: ${ }^{*} \mathrm{P}<0.05,{ }^{* *} \mathrm{P}<0.01$; comparison with the control group: ${ }^{\# \#} \mathrm{P}<0.01$. Cyt.c, cytochrome $c$.

that the promotion of angiogenesis exhibits anti-osteoporotic effects $(6,7,9)$. Naringin, the main active ingredient of the traditional Chinese medicinal fern Drynaria, is characterized by estrogen-like properties that exhibit therapeutic effects on OP by a mechanism that has not been elucidated $(16,20)$. Numerous studies have shown that naringin promotes cell proliferation and inhibits cell apoptosis $(16-18,20,23,24)$. However, its effects on VEC apoptosis and function, as well as on angiogenesis, have not been reported. This study showed, for the first time, that naringin exhibits a significant proliferation-promoting effect on VECs in a time- and dose-dependent manner. Previous studies of the induction of endothelial cell apoptosis in vitro mostly used exogenous apoptosis-inducing agents (such as hydrogen peroxide) to construct an apoptosis model (25), a method which may not accurately reflect in vivo conditions. Therefore, in this study, we employed a serum starvation-induced model of apoptosis to simulate an in vivo-like pathological state of nutritional deficiency in VECs $(26,27)$. Our results showed that we successfully induced apoptotic cell death by serum starvation, according to the cellular and nuclear morphological changes observed in VECs, similar to previous studies $(26,27)$. Previous studies found that naringin inhibited apoptosis in human neuroblastoma cells and neurons $(23,24)$, further supporting our findings that the apoptosis ratio was significantly decreased in both the high- and low-concentration naringin treatment groups compared to that in the serum starvation group. These results indicated that naringin reduced the serum starvation-induced apoptosis ratio in VECs, but was incapable of completely inhibiting apoptosis.

There are two classical apoptotic pathways: the death receptor pathway and the mitochondrial-mediated pathway, of which the former recruits and activates primarily caspase- 8 through the binding of the Fas ligand on the cell membrane to its receptor, leading to cell degradation. Mitochondrial permeability transition results in the reduction in mitochondrial membrane potential and release of Cyt.c into the cytoplasm, activating caspase- 9 to cause apoptosis via the mitochondrial-mediated pathway (28). In the newly discovered ER stress-mediated apoptotic pathway, severe ER stress leads to the upregulation of proteins such as GRP78, CHOP, and caspase-12, leading to apoptosis (29). Caspase-3 is the central component of apoptotic pathways. Our results showed that the increase in apoptosis ratio induced by serum starvation was correlated with increased expression of Cyt.c, 

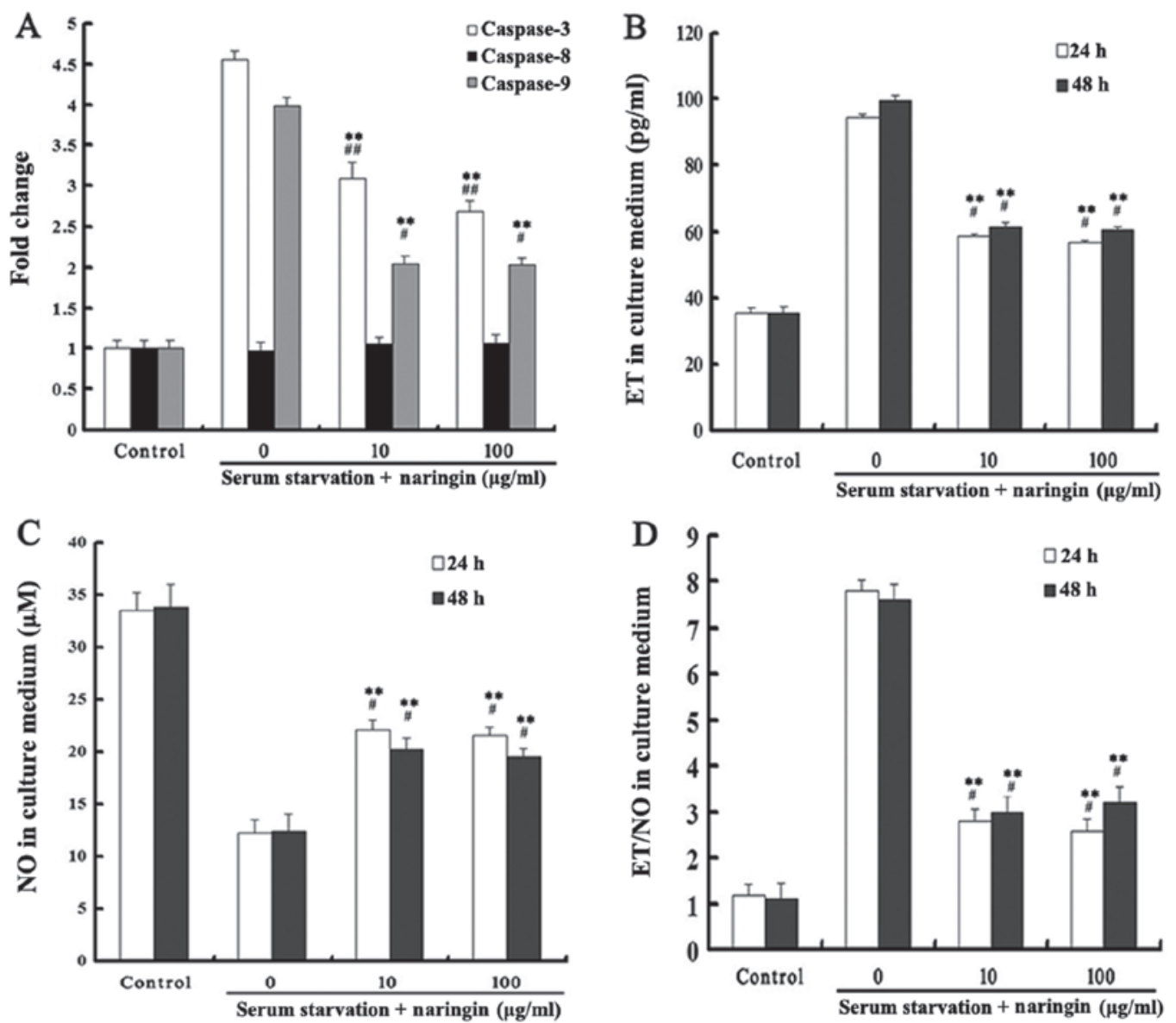

Figure 5. Effects of naringin on the expression of caspase-3, caspase-8, caspase-9, ET, and NO. (A) Naringin inhibited the serum starvation-induced increase in caspase-3 and -9 activities, but had no effect on caspase-8. (B-D) Naringin regulated the ET/NO ratio by inhibiting the expression of ET while enhancing the expression of NO. Comparison with the serum starvation group: ${ }^{* *} \mathrm{P}<0.01$; comparison with the control group: ${ }^{\#} \mathrm{P}<0.05,{ }^{\# \#} \mathrm{P}<0.01$. ET, endothelin; NO, nitric oxide.
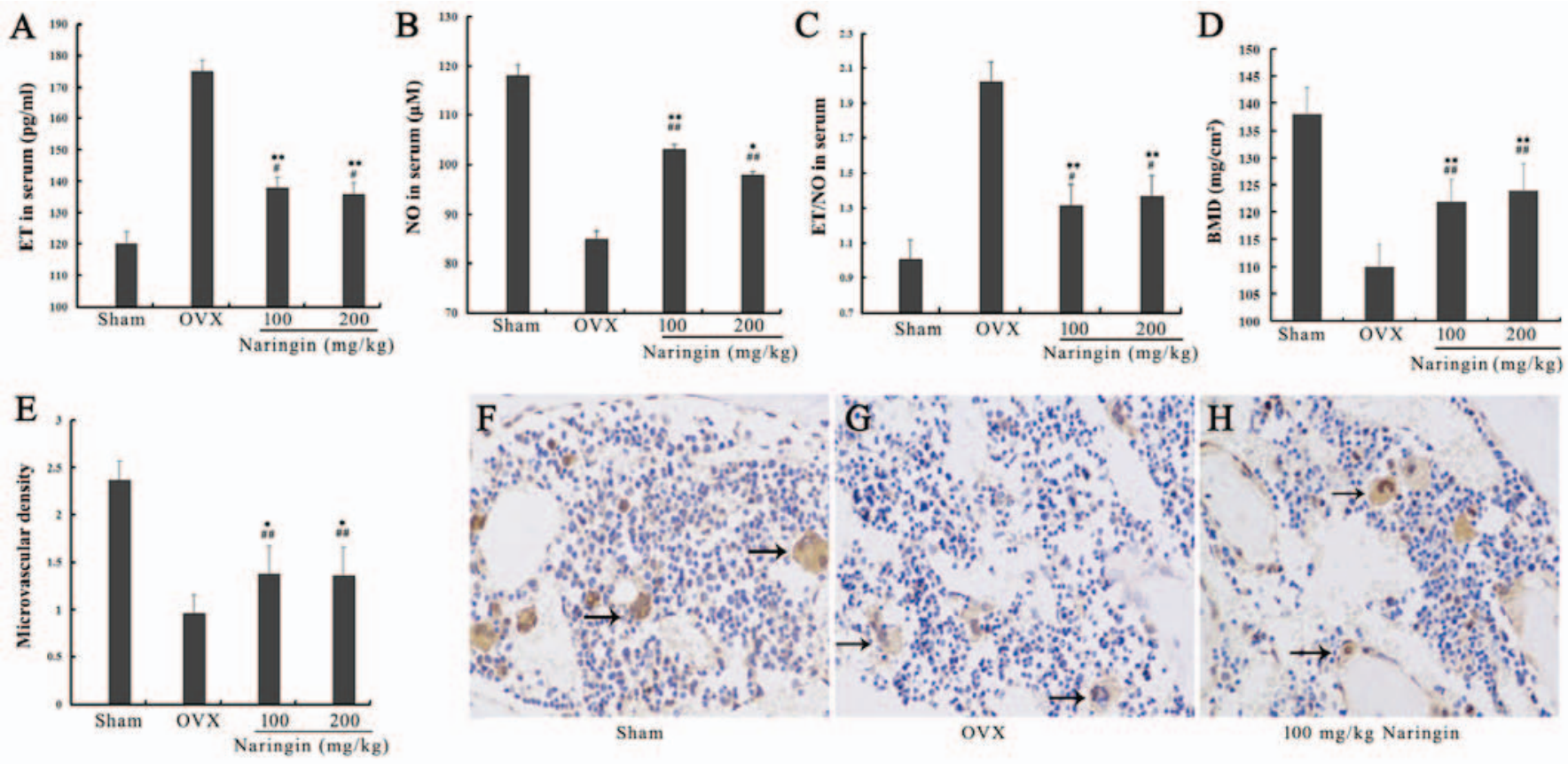

Figure 6. Effects of naringin on the expression of ET and NO in rat serum, and on BMD and microvessel angiogenesis. (A-C) Naringin regulated the ET/NO ratio by inhibiting the expression of ET while enhancing the expression of NO in serum. (D) Naringin partially inhibited the OVX-induced reduction in BMD in rats. (E) Microvascular density analysis showed that naringin promoted bone marrow microvascular density in OVX rats. (F-H) Images showing CD43 staining of bone marrow microvessels at the distal femur. Naringin significantly inhibited the OVX-induced reduction in bone marrow microvessels (as indicated by arrows); original magnification, $\mathrm{x} 200$. Comparison with the OVX group: ${ }^{*} \mathrm{P}<0.05,{ }^{* *} \mathrm{P}<0.01$; comparison with the sham group: ${ }^{*} \mathrm{P}<0.05$, ${ }^{\# \#} \mathrm{P}<0.01$. $\mathrm{ET}$, endothelin; NO, nitric oxide; BMD, bone mineral density; OVX, ovariectomized. 
caspase-9, and proteins associated with ER stress (GRP78, CHOP, and caspase-12), and decreased mitochondrial membrane potential. These findings indicated that serum starvation induced apoptosis in VECs via ER stress- and mitochondrial-mediated pathways. However, the expression of caspase- 8 was unaffected by this process, suggesting that the death receptor-mediated pathway of apoptosis was not involved. Intervention with both high and low concentrations of naringin inhibited the expression of GRP78, CHOP, Cyt.c, and caspases $-3,-9$, and -12 as well as decreased the mitochondrial membrane potential, thereby reducing the apoptosis ratio. These results preliminarily indicate that naringin inhibits starvation-induced apoptosis in VECs by inhibiting the mitochondrial- and ER stress-mediated apoptotic pathways. These findings are similar to results reported by Kim et al (23), who showed that naringin inhibited apoptosis induced by rotenone in human neuroblastoma cells by reducing caspase-3 and -9 activity. However, we found that the inhibitory effect of naringin on apoptosis did not reach the level of the control group, suggesting other apoptotic pathways may participate in starvation-induced apoptosis in VECs in addition to the ER stress- and mitochondrial-mediated pathways. Estrogen receptor activation can inhibit apoptosis in VECs by regulating the ER stress-mediated pathway (30), and some studies have shown that naringin has estrogen-like effects $(16,31)$. Whether these effects play a role in inhibiting apoptosis, however, requires verification by further studies.

Vascular endothelium can produce and release various vasoactive substances after injury, the most important of which are ET and NO (32). ET exerts a significant vasoconstrictive effect, and studies have found that serum levels of ET in postmenopausal OP patients were significantly decreased after hormone replacement therapy with estrogen, suggesting that ET can affect bone metabolism $(33,34)$. NO is an endothelium-derived relaxing factor (EDRF) with multiple beneficial effects such as vasodilation, inhibition of oxygen-derived free radicals, and prevention of leukocyte adhesion to vessel walls. Studies have shown that naringin can regulate the synthesis of NO, thus improving Huntington's disease-like symptoms in rats (35) and post-stroke depression in mice via NO modulation (36). Recent studies have demonstrated that ET/NO are important vasoconstrictory/vasodilatory factors and that disruption of their balance leads to microcirculatory disturbances, affecting tissue metabolism (32). Therefore, some studies have suggested that ET/NO disruption directly affects bone microcirculation, preventing blood nutrients from being transported into bone tissue and thereby affecting bone metabolism, contributing to the pathogenesis of OP (9). Our in vitro experiments showed that both low and high concentrations of naringin reduced the ET/NO ratio in culture medium by inhibiting the excretion of ET by endothelial cells while enhancing NO expression. We further tested naringin in the OVX rat model of OP and found that it exerts a similar in vivo and in vitro regulatory function on $\mathrm{ET}$ and $\mathrm{NO}$, and is associated with BMD in rats. These results indicate that naringin allows VECs to function normally by regulating ET and NO expression and maintaining their balance in vivo and in vitro, a mechanism by which naringin may modulate vascular function to further affect bone metabolism.
Studies suggest that localized nutritional deficiency in osteoblasts due to vascular dysfunction and reduced neovascularization contributes to the pathogenesis of OP, and that modulation of angiogenesis can be beneficial in patients with OP $(2,7,9)$. Recent studies have found that OVX mice with OP have a significantly reduced distribution of intraosseous blood vessels compared with control mice, indicating that localized intraosseous angiogenesis is significantly associated with OP (8). In addition, some researchers have reviewed the correlation between OP and blood vessels from different perspectives and have suggested that aging-induced vascular dysfunction may be associated with OP; hence, the control of angiogenesis can reduce risk factors for $\mathrm{OP}$ as well as incidence and mortality rates for OP-related diseases $(9,37)$. The OVX model also promotes apoptosis and bone resorption while inhibiting angiogenesis and bone development (38), findings that have indirectly confirmed the correlation between localized angiogenesis and OP. After we treated OVX rats with naringin for 3 months, MVD analysis in the distal femur showed that the treatment groups had significantly higher MVD than the OVX group, and were positively correlated with BMD. Preliminary results indicate that the promotion of angiogenesis may be one mechanism by which naringin exerts its anti-osteoporotic effects.

We showed that naringin promoted VEC proliferation and inhibited serum starvation-induced apoptosis in VECs by inhibiting the mitochondrial- and ER stress-mediated apoptotic pathways. Additionally, it regulated the function of endothelial cells and promoted angiogenesis, thereby exhibiting an anti-osteoporotic effect. This study provided experimental evidence to clarify the mechanism of action of naringin in the treatment of OP.

\section{Acknowledgements}

This study was supported by grants from the National Natural Science Foundation of China (81403443), Shanghai Municipal Commission of Health and Family Planning (no. 2014Y039 and 'Xing-Lin New Star').

\section{References}

1. Lane NE: Epidemiology, etiology, and diagnosis of osteoporosis. Am J Obstet Gynecol 194 (Suppl): S3-S11, 2006.

2. Almubarak S, Nethercott H, Freeberg M, Beaudon C, Jha A, Jackson W, Marcucio R, Miclau T, Healy K and Bahney C: Tissue engineering strategies for promoting vascularized bone regeneration. Bone 83: 197-209, 2016.

3. Street J, Bao M, deGuzman L, Bunting S, Peale FV Jr, Ferrara N, Steinmetz H, Hoeffel J, Cleland JL, Daugherty A, et al: Vascular endothelial growth factor stimulates bone repair by promoting angiogenesis and bone turnover. Proc Natl Acad Sci USA 99: 9656-9661, 2002.

4. Shen X, Wan C, Ramaswamy G, Mavalli M, Wang Y, Duvall CL, Deng LF, Guldberg RE, Eberhart A, Clemens TL, et al: Prolyl hydroxylase inhibitors increase neoangiogenesis and callus formation following femur fracture in mice. J Orthop Res 27: 1298-1305, 2009

5. Song HJ, Lan BS, Cheng B, Zhang KF, Yan HW, Wang WZ and Gao ZQ: Treatment of early avascular necrosis of femoral head by small intestinal submucosal matrix with peripheral blood stem cells. Transplant Proc 43: 2027-2032, 2011.

6. Liu XD, Cai F, Liu L, Zhang Y and Yang AL: MicroRNA-210 is involved in the regulation of postmenopausal osteoporosis through promotion of VEGF expression and osteoblast differentiation. Biol Chem 396: 339-347, 2015. 
7. Saran U, Gemini Piperni S and Chatterjee S: Role of angiogenesis in bone repair. Arch Biochem Biophys 561: 109-117, 2014.

8. Ding WG, Wei ZX and Liu JB: Reduced local blood supply to the tibial metaphysis is associated with ovariectomy-induced osteoporosis in mice. Connect Tissue Res 52: 25-29, 2011.

9. Alagiakrishnan K, Juby A, Hanley D, Tymchak W and Sclater A: Role of vascular factors in osteoporosis. J Gerontol A Biol Sci Med Sci 58: 362-366, 2003.

10. Peng J, Hui K, Hao C, Peng Z, Gao QX, Jin Q, Lei G, Min J, Qi Z, Bo C, et al: Low bone turnover and reduced angiogenesis in streptozotocin-induced osteoporotic mice. Connect Tissue Res 57: 277-289, 2016.

11. Scull CM and Tabas I: Mechanisms of ER stress-induced apoptosis in atherosclerosis. Arterioscler Thromb Vasc Biol 31: 2792-2797, 2011

12. Smith MA and Schnellmann RG: Calpains, mitochondria, and apoptosis. Cardiovasc Res 96: 32-37, 2012.

13. Prisby RD, Ramsey MW, Behnke BJ, Dominguez JM 2nd, Donato AJ, Allen MR and Delp MD: Aging reduces skeletal blood flow, endothelium-dependent vasodilation, and NO bioavailability in rats. J Bone Miner Res 22: 1280-1288, 2007.

14. Dominguez JM II, Prisby RD, Muller-Delp JM, Allen MR and Delp MD: Increased nitric oxide-mediated vasodilation of bone resistance arteries is associated with increased trabecular bone volume after endurance training in rats. Bone 46: 813-819, 2010

15. Bharti S, Rani N, Krishnamurthy B and Arya DS: Preclinical evidence for the pharmacological actions of naringin: A review. Planta Med 80: 437-451, 2014.

16. Pang WY, Wang XL, Mok SK, Lai WP, Chow HK, Leung PC, Yao XS and Wong MS: Naringin improves bone properties in ovariectomized mice and exerts oestrogen-like activities in rat osteoblast-like (UMR-106) cells. Br J Pharmacol 159: 1693-1703, 2010.

17. Liu M, Li Y and Yang ST: Effects of naringin on the proliferation and osteogenic differentiation of human amniotic fluid-derived stem cells. J Tissue Eng Regen Med n/a, 2014.

18. Zhang P, Dai KR, Yan SG, Yan WQ, Zhang C, Chen DQ, $\mathrm{Xu} \mathrm{B}$ and $\mathrm{Xu} \mathrm{ZW}$ : Effects of naringin on the proliferation and osteogenic differentiation of human bone mesenchymal stem cell. Eur J Pharmacol 607: 1-5, 2009.

19. Wei M, Yang Z, Li P, Zhang Y and Sse WC: Anti-osteoporosis activity of naringin in the retinoic acid-induced osteoporosis model. Am J Chin Med 35: 663-667, 2007.

20. Li F, Sun X, Ma J, Ma X, Zhao B, Zhang Y, Tian P, Li Y and Han Z: Naringin prevents ovariectomy-induced osteoporosis and promotes osteoclasts apoptosis through the mitochondria-mediated apoptosis pathway. Biochem Biophys Res Commun 452: 629-635, 2014.

21. Zhang YH,Zhao CQ, Jiang LS and Dai LY: Cyclic stretch-induced apoptosis in rat annulus fibrosus cells is mediated in part by endoplasmic reticulum stress through nitric oxide production. Eur Spine J 20: 1233-1243, 2011.

22. Padró T, Ruiz S, Bieker R, Bürger H, Steins M, Kienast J, Büchner T, Berdel WE and Mesters RM: Increased angiogenesis in the bone marrow of patients with acute myeloid leukemia. Blood 95: 2637-2644, 2000.

23. Kim HJ, Song JY, Park HJ, Park HK, Yun DH and Chung JH: Naringin protects against rotenone-induced apoptosis in human neuroblastoma SH-SY5Y cells. Korean J Physiol Pharmacol 13: 281-285, 2009
24. Rong W, Wang J, Liu X, Jiang L, Wei F, Hu X, Han X and Liu Z: Naringin treatment improves functional recovery by increasing BDNF and VEGF expression, inhibiting neuronal apoptosis after spinal cord injury. Neurochem Res 37: 1615-1623, 2012

25. Liu XX, Tang L, Ge R, Li JK, Kang Y, Zhu MX, Li QS and Hao XL: iTRAQ-based quantitative proteomic analysis of the anti-apoptotic effect of hyperin, which is mediated by Mcl-1 and Bid, in $\mathrm{H}_{2} \mathrm{O}_{2}$-injured EA.hy926 cells. Int J Mol Med 37: 1083-1090, 2016.

26. Date T, Taniguchi I, Inada K, Matsuo S, Miyanaga S, Yamane T, Abe Y, Sugimoto K and Mochizuki S: Nicorandil inhibits serum starvation-induced apoptosis in vascular endothelial cells. J Cardiovasc Pharmacol 46: 721-726, 2005.

27. Hogg N, Browning J, Howard T, Winterford C, Fitzpatrick D and Gobé G: Apoptosis in vascular endothelial cells caused by serum deprivation, oxidative stress and transforming growth factor-beta. Endothelium 7: 35-49, 1999.

28. Hengartner MO: The biochemistry of apoptosis. Nature 407: 770-776, 2000

29. Holcik M and Sonenberg N: Translational control in stress and apoptosis. Nat Rev Mol Cell Biol 6: 318-327, 2005

30. Yu J, Eto M, Akishita M, Okabe T and Ouchi Y: A selective estrogen receptor modulator inhibits TNF-alpha-induced apoptosis by activating ERK1/2 signaling pathway in vascular endothelial cells. Vascul Pharmacol 51: 21-28, 2009.

31 . Wong RW and Rabie AB: Effect of naringin collagen graft on bone formation. Biomaterials 27: 1824-1831, 2006.

32. Uhlmann D, Uhlmann S and Spiegel HU: Endothelin/nitric oxide balance influences hepatic ischemia-reperfusion injury. J Cardiovasc Pharmacol 36 (Suppl 1): S212-S214, 2000.

33. Haenggi W, Bersinger NA, Mueller MD, and Birkhaeuser MH: Decrease of serum endothelin levels with postmenopausal hormone replacement therapy or tibolone. Gynecol Endocrinol 13: 202-205, 1999

34. Gulhan I, Kebapcilar L, Alacacioglu A, Bilgili S, Kume T, Aytac B and Gunaydin R: Postmenopausal women with osteoporosis may be associated with high endothelin-1. Gynecol Endocrinol 25: 674-678, 2009.

35. Kumar P and Kumar A: Protective effect of hesperidin and naringin against 3-nitropropionic acid induced Huntington's like symptoms in rats: Possible role of nitric oxide. Behav Brain Res 206: 38-46, 2010.

36. Aggarwal A, Gaur V and Kumar A: Nitric oxide mechanism in the protective effect of naringin against post-stroke depression (PSD) in mice. Life Sci 86: 928-935, 2010.

37. Byon $\mathrm{CH}$ and Chen Y: Molecular mechanisms of vascular calcification in chronic kidney disease: The link between bone and the vasculature. Curr Osteoporos Rep 13: 206-215, 2015.

38. Orlić I, Borovecki F, Simić P and Vukicević S: Gene expression profiling in bone tissue of osteoporotic mice. Arh Hig Rada Toksikol 58: 3-11, 2007.

This work is licensed under a Creative Commons Attribution-NonCommercial-NoDerivatives 4.0 International (CC BY-NC-ND 4.0) License. 